\title{
PRESUNCIÓN DE INOCENCIA Y CONCEPCIONES DE LA OBLIGACIÓN MORAL
}

\author{
Presumption of Innocence and \\ Conceptions of Moral Obligation
}

\author{
Hugo Omar Seleme* \\ Universidad Nacional de Córdoba \\ Córdoba, Argentina
}

RESUMEN: El presente trabajo intenta mostrar, en primer lugar, que el modo de concebir el principio de inocencia depende de la concepción de obligación moral que se posea. Esta vinculación entre ambas concepciones -la de obligación y la del principio de inocencia- no ha sido explorada en la literatura especializada. El trabajo intenta corregir este déficit. En segundo lugar, y una vez establecida la vinculación entre ambas concepciones, el trabajo se encarga de mostrar que el modo correcto de concebir la obligación moral es uno de índole prospectivista. Esto permite extraer la conclusión de que la manera correcta de concebir el principio de inocencia es la correlacionada con dicha concepción de obligación moral.

PALABRAS CLAVE: prueba, castigo, verdad

Investigador del CONICET. Catedrático de Ética de la Facultad de Derecho de la Universidad Nacional de Córdoba. Correo electrónico: <hugoseleme@gmail.com>.

Artículo recibido el 26 de diciembre de 2016 y aceptado para publicación el 15 de marzo de 2017. 
SELEME, HUGO OMAR: PRESUNCIÓN DE INOCENCIA Y CONCEPCIONES DE LA OBLIGACIÓN MORAL

ABSTRACT: The present paper pursues two objectives. First, it tries to show that the way of conceiving the Principle of Innocence depends on the conception of moral obligation that is adopted. This linkage between both conceptions has not been explored in the specialized literature. The paper attempts to correct this deficit. Secondly, and once the link between both conceptions is established, it is argued for a particular conception of moral obligation. Specifically, several reasons are offered in favor of the prospective variant. Finally, this leads to the conclusion that the correct conception of the Principle of Innocence is that correlated with the prospective conception of moral obligation.

KEYWORDS: proof, punishment, truth

\section{INTRODUCCIÓN}

El principio de inocencia ocupa un lugar destacado en el proceso penal de países que poseen sistemas jurídicos que se inspiran en diferentes tradiciones legales. En Chile, el principio ha sido incorporado en el artículo 4 del Código Procesal Penal, según el cual "ninguna persona será considerada culpable ni tratada como tal en tanto no fuere condenada por una sentencia firme". En Argentina, antes de la reforma constitucional del año 1994, el principio era extraído del artículo 18, según el cual: "Ningún habitante de la Nación puede ser penado sin juicio previo fundado en ley anterior al hecho del proceso, ni juzgado por comisiones especiales, o sacado de los jueces designados por la ley antes del hecho de la causa. Nadie puede ser obligado a declarar contra sí mismo; ni arrestado sino en virtud de orden escrita de autoridad competente". Luego de la reforma, con la incorporación de los tratados y convenciones de derechos humanos, establecida por el artículo 75 inciso 22, el principio posee reconocimiento expreso. Esto se debe a la incorporación de la Declaración Universal de los Derechos Humanos, cuyo artículo 11 inciso 1 establece: "Toda persona acusada de delito tiene derecho a que se presuma su inocencia mientras no se pruebe su culpabilidad, conforme a la ley y en juicio público en el que se le hayan asegurado todas las garantías necesarias para su defensa". El mismo principio también está reconocido en otros instrumentos internacionales con jerarquía constitucional, como la Declaración Americana de los Derechos y Deberes 
del Hombre1, la Convención Americana sobre Derechos Humanos² y el Pacto Internacional de Derechos Civiles y Políticos³.

El principio también ha sido incorporado recientemente a la Constitución española, la cual en su artículo 24 inciso 2 establece que "todos tienen derecho al Juez ordinario predeterminado por la ley, a la defensa y a la asistencia de letrado, a ser informados de la acusación formulada contra ellos, a un proceso público sin dilaciones indebidas y con todas las garantías, a utilizar los medios de prueba pertinentes para su defensa, a no declarar contra sí mismos, a no confesarse culpables y a la presunción de inocencia". En los Estados Unidos el principio ha sido reconocido en numerosos fallos judiciales, siendo el más relevante Coffin v. United States, en el cual se señala que "el principio de que existe una presunción de inocencia en favor del acusado es la indubitada ley, axiomática y elemental". ${ }^{4}$ Por lo que respecta a instrumentos internacionales regionales, la Convención Europea de Derechos Humanos indica en su artículo 6 inciso 2: "Cualquier acusado de un delito penal será presumido inocente hasta que se pruebe en un proceso legal que es culpable".

La dogmática también se ha ocupado -profusamente- de intentar encontrar el alcance exacto del principio. Así, para Cardenas Rioseco "la presunción de inocencia es un derecho subjetivo público, que se ha elevado a la categoría de derecho humano fundamental que posee su eficacia en un doble plano: por una parte, opera en las situaciones extraprocesales y constituye el derecho a recibir la consideración y el trato de no autor o no partícipe en hechos de carácter delictivo o análogos a éstos; por otro lado, el referido derecho opera fundamentalmente en el campo procesal, con influjo decisivo en el régimen jurídico de la prueba". ${ }^{5}$ Manzini Vicenzo, por su parte, considera que el principio establece que la calidad de no-culpable es inherente a la calidad de persona humana. ${ }^{6}$ Para Maier, el principio impide otorgar el trato de culpable a cualquier persona, sea cual sea el grado de verosimilitud de la acusación, mientras el Estado a través de sus órganos judiciales no haya declarado

1 El artículo 26, en su primera parte, señala: "Se presume que todo acusado es inocente, hasta que se pruebe que es culpable".

2 El artículo 8 inciso 2, sostiene: "Toda persona inculpada de delito tiene derecho a que se presuma su inocencia mientras no se establezca legalmente su culpabilidad".

3 El artículo 14 inciso 2, declara: "Toda persona acusada de un delito tiene derecho a que se presuma su inocencia mientras no se pruebe su culpabilidad conforme a la ley".

4 Corte Suprema de Estados Unidos, Coffin v. United States (1895) p. 453.

5 CÁRdenas (2006) p. 23.

6 MANZINI (1951) p. 180. 

CIÓN MORAL

su culpabilidad. ${ }^{7}$ Martínez Remigio sostiene que el principio no solo se aplica a la fase del juicio oral, sino también al proceso de investigación y a la tarea de evaluación de la prueba por parte del Tribunal. Como consecuencia, deben restringirse al mínimo las medidas que reducen derechos durante el proceso, la carga de la prueba cae sobre quien acusa, y debe absolverse si la prueba no es suficiente. ${ }^{8}$ Nogueira Alcalá, por su parte, lo trata como un principio omnicomprensivo que informa todo el proceso y permite distinguir los sistemas acusatorios, fundados en el principio de inocencia, y los sistemas inquisitivos, donde el inculpado es tratado como si fuese culpable. ${ }^{9}$

Quizás por el rango de las normas en las que el principio es expuesto, tratados internacionales y textos constitucionales, por la pluralidad de sistemas jurídicos de los que forma parte, o por el número creciente de dogmáticos que han focalizado su atención en el mismo, ha existido una proliferación de ámbitos en los que el principio de inocencia se considera aplicable. Tradicionalmente se entiende que el principio tiene aplicaciones tanto procesales como extraprocesales. Con referencia a estas últimas se sostiene que ni los órganos gubernamentales - diferentes a los involucrados en el proceso judicial- ni los particulares pueden conferir a ningún ciudadano el trato de un autor del delito, hasta tanto esto no haya sido establecido en un proceso judicial. Así, por ejemplo, los medios de comunicación no pueden afirmar que un ciudadano es culpable de un delito si esto no ha sido determinado judicialmente.

Dada esta pluralidad de funciones que se atribuyen al principio, cualquier trabajo que se dedique a investigarlo debe, en primer lugar, aclarar cuál es la faceta del mismo en la que pretende concentrarse. Esto no implica negar, ni tampoco afirmar, que el principio cumple las funciones adicionales que se le atribuyen. Simplemente se trata de una decisión metodológica para circunscribir el objeto de estudio. En lo que sigue solo estaré interesado en la particular función que el principio cumple en relación con la tarea de evaluar los hechos en el seno del proceso judicial.

La hipótesis del presente trabajo es, en primer lugar, que el modo de concebir el principio depende de la concepción de obligación que se posea. Esta vinculación entre ambas concepciones -la de obligación y la del principio de inocencia - no ha sido explorada en la literatura especializada a pesar de que, como intentaré mostrar, las implicaciones que una tiene sobre la otra

\footnotetext{
MAIER (1996) p. 490.

Martínez (2005) p. 25.

NOGUeira (2005).
} 
son profundas. En segundo lugar, y una vez establecida la vinculación entre ambas concepciones, el trabajo se encarga de mostrar que el modo correcto de concebir la obligación moral es uno de índole prospectivista. Esto permite extraer la conclusión de que la manera correcta de concebir el principio de inocencia es la correlacionada con dicha concepción de obligación moral.

\section{CONCEPCIONES DE OBLIGACIÓN Y EL DEBER DE CASTIGAR}

El principio de inocencia, como regla para evaluar los hechos en el proceso penal, se encuentra directamente vinculado con el ejercicio de la coacción estatal. A los fines de explorar el sentido del Principio de Inocencia, por lo tanto, es indispensable adoptar alguna posición sobre la justificación del castigo impuesto por el Estado. La premisa sobre la que fundaré mi argumento es una según la cual el Estado legítimo no es solo aquel que tiene permitido el ejercicio de la coacción, entendiendo por esto que sus ciudadanos no tienen derecho a no ser $\operatorname{coaccionados}^{10}$. El Estado legítimo, en ciertas circunstancias, tiene el deber de coaccionar. Específicamente, el Estado tiene la obligación moral de imponer los castigos respetando lo establecido por la norma penal sustantiva. ${ }^{11}$

Una consecuencia que se sigue de esta premisa es que el mejor curso de acción que un Estado puede adoptar - a la hora de administrar el castigo- es el de condenar a todos los culpables de haber transgredido la norma penal y absolver a todos los inocentes. Si la norma sustantiva penal es justa -tal como presupondré a los fines del presente trabajo- y la condena a quien la trasgrede se encuentra justificada, entonces este curso de acción es moralmente superior a aquel en el que el Estado condena solo a individuos culpables, pero no a todos ellos -por ejemplo, solo a los que pertenecen a cierto estrato social-, o no condena a individuos inocentes, pero tampoco a individuos culpables.

Usualmente se piensa que la obligación estatal de administrar el castigo respetando lo establecido por la norma penal sustantiva implica que el Estado debe condenar al culpable de haberla transgredido y absolver al inocente. Según esta reconstrucción el Estado tiene la obligación moral de seguir el mejor curso de acción, el que a su vez se determina a partir de lo que establece

10 Entre quienes entienden a la legitimidad política de esta manera se encuentra LADENSON (1980).

11 En lo que sigue daré por sentado que el castigo penal se encuentra moralmente justificado y que la norma sustantiva que fija el castigo lo hace de manera moralmente correcta. Por supuesto que estos son temas controvertidos, pero exceden el objetivo específico de este trabajo. 
la norma penal sustantiva. Así, por ejemplo, si la norma penal señala que quien mata debe ser condenado, el mejor curso de acción es aquel en donde los que efectivamente han transgredido la prohibición, y solo ellos, son condenados. A su vez, el Estado satisface su obligación de administrar el castigo respetando la norma penal sustantiva solo si condena al culpable y absuelve al inocente.

En esta reconstrucción cada vez que el Estado condena a un inocente o absuelve a un culpable transgrede su obligación moral. En este supuesto a lo más que puede aspirar el Estado es a excusarse de la transgresión. El modo habitual de hacerlo es señalar que el proceso judicial de determinación de los hechos no es infalible y a lo más que puede aspirar es a establecer los hechos con un cierto grado de certeza a partir de la prueba ofrecida. Si, contrario a la prueba ofrecida en la causa, los hechos son falsos, el Estado puede argüir ignorancia como excusa para la transgresión de su obligación.

A pesar de que esta reconstrucción de la obligación estatal aparece como plausible y hasta inevitable, creo que ninguna de las dos cosas son ciertas. Como intentaré mostrar en el apartado siguiente, la reconstrucción es incorrecta y, como espero dejar claro a continuación, no es inevitable dado que existen reconstrucciones alternativas. La razón de que esto sea así es que existen al menos tres maneras de concebir la obligación moral y, por ende, tres maneras diferentes de concebir la obligación moral del Estado de administrar el castigo respetando lo establecido por la norma penal sustantiva. La reconstrucción habitual que he presentado utiliza una de estas concepciones, quedando por explorar las dos restantes.

La concepción de obligación moral utilizada en la reconstrucción habitual de la obligación estatal de castigar es una de carácter objetivista, según la cual un agente debe realizar un acto si, y solo si, es la mejor opción que él o ella tiene. ${ }^{12}$ En consecuencia, un agente ha cumplido su obligación moral si no existe ningún curso de acción que sea mejor que el que de hecho adoptó. Es moralmente incorrecto para un agente realizar un acto si existía un curso de acción alternativo mejor. ${ }^{13}$ Es esta concepción de obligación la que

12 La concepción objetivista de obligación ha sido aceptada, entre otros, por MOORE (1912).

13 Las diferentes concepciones objetivas de obligación se distinguen por el criterio sustantivo que utilizan a la hora de determinar que un curso de acción es mejor que otro. Así, una concepción objetivista de obligación que utilice como criterio el consecuencialismo del acto sostendrá que el mejor curso de acción es aquel que efectivamente tiene por resultado maximizar el monto de aquello que se considera intrínsecamente valioso. Una concepción que utilice como criterio el consecuencialismo de la regla sostendrá que el 
permite concluir que si el mejor curso de acción que puede adoptar el Estado a la hora de administrar las condenas es imponerlas sobre todos y cada uno de los individuos culpables y no imponerlas nunca sobre un inocente, entonces el Estado está obligado a condenar al culpable y tiene prohibido condenar a inocentes, o lo que es lo mismo, está obligado a no condenar inocentes.

Una concepción alternativa de obligación moral es aquella según la cual un agente debe realizar un acto si, y solo si, él cree que es la mejor opción que él o ella tiene. ${ }^{14}$ De acuerdo con esta concepción de obligación moral alguien cumple con su obligación cuando sigue el que cree que es el mejor curso de acción, con independencia de que de hecho efectivamente lo sea. Tampoco importa el carácter de la evidencia que respalda su creencia. Basta que crea que es el mejor curso de acción, a pesar de que lo haga sobre la base de una evidencia insuficiente o defectuosa, para que exista la obligación moral de seguirlo. ${ }^{15}$

Si uno posee esta concepción subjetiva de obligación moral, la obligación estatal de castigar y absolver respetando la norma penal sustantiva adquiere contornos diferentes. Aunque el mejor curso de acción sigue siendo condenar a los transgresores y absolver a los inocentes, el Estado ahora no tiene la obligación moral de seguir el mejor curso de acción. Por el contrario, su obligación es ahora seguir el que cree que es el mejor curso de acción. Mientras que de acuerdo con la versión habitual lo que importa es la verdad acerca de los hechos ventilados en el proceso judicial, lo que importa de acuerdo con esta nueva reconstrucción son las creencias de quien tiene la potestad para fijar los hechos dentro del proceso. Si el Estado administra el castigo a aquellos que quien tiene esta potestad cree culpables y absuelve a quienes se cree son inocentes, entonces ha cumplido su obligación moral.

mejor curso de acción es aquel acorde con una regla cuyo seguimiento generalizado produciría la efectiva maximización de la cualidad considerada intrínsecamente valiosa. Una concepción que utilice un criterio que no hace referencias a las consecuencias de los cursos de acción sino a su carácter virtuoso afirmará que el mejor curso de acción es aquel que efectivamente muestra el mayor grado de virtud, sea como sea que se la conciba. Si la concepción utiliza como criterio el respeto por los derechos sostendrá que el mejor curso de acción es aquel que efectivamente satisface o no vulnera estos derechos. ZIMMERMAN (2008) pp. 3 y 4.

14 ZimMERMAn (2008) p. 3.

15 La concepción subjetivista de obligación fue adoptada, entre otros, por PRICHARD (1949) y Ross (1930, 1939). 
La ignorancia sobre los hechos a la hora de aplicar el castigo no aparece ya como una excusa, como sucede si uno adopta la concepción objetivista de obligación, sino que altera los términos de la obligación estatal. Si el juzgador sobre los hechos ignora que la persona condenada es inocente, esto es cree que es culpable, el Estado ha cumplido con su obligación al castigarla. Lo mismo si absuelve al culpable que el juzgador sobre los hechos cree inocente. Lo que fija los contornos de la obligación estatal de castigar y absolver no son más los hechos, sino la íntima convicción del juzgador. El carácter falible del proceso judicial -que determina que las certezas a las que arriba el juzgador no coincidan necesariamente con la realidad- no funciona como una excusa para que el Estado se exima del reproche por no haber cumplido con su obligación moral, sino que altera los términos de la obligación que posee.

Finalmente, existe una tercera concepción de obligación moral que puede adoptarse para reconstruir la obligación estatal de castigar y absolver. Según esta concepción un agente debe realizar un acto si no existe otro que posea un mayor valor esperado, dada la evidencia disponible ponderada por su grado de confiabilidad. ${ }^{16}$

16 La concepción prospectiva de obligación es más compleja que como aquí se la presenta. En su formulación completa ZIMMERMAN (2008) p. 78 la presenta de la siguiente manera: "Para cualquier acto, A;

(1) A es prima facie moralmente correcto si y solo si existe algún aspecto, S, de la situación del agente tal que ninguna otra opción que tiene le ofrece un mejor prospecto de hacer lo que es adecuado para $S$.

(2) A es prima facie moralmente incorrecto si y solo si existe algún aspecto, S, de la situación del agente tal que alguna otra opción que tiene le ofrece un mejor prospecto de hacer lo que es adecuado para S;

(3) A es prima facie moralmente obligatorio si y solo si existe algún aspecto, S, de la situación del agente tal que $\mathrm{A}$ le ofrece un prospecto de hacer lo que es adecuado para $\mathrm{S}$ mejor que el provisto por cualquier otra opción que tenga.

(4) A es moralmente correcto todo considerado si y solo si ninguna otra opción que el agente tiene le ofrece un mejor prospecto de hacer lo que es adecuado para su situación como un todo.

(5) A es moralmente incorrecto todo considerado si y solo si alguna otra opción que el agente tiene le ofrece un mejor prospecto de hacer lo que es adecuado para su situación como un todo.

(6) A es moralmente obligatorio todo considerado si y solo si A le ofrece al agente un prospecto de hacer lo que es adecuado para su situación como un todo mejor que el ofrecido por cualquier otra opción que tenga". 
Michael Zimmerman, quien ha identificado y defendido esta tercera concepción de obligación, denomina a este curso de acción el prospectivamente mejor. Que algo sea prospectivamente mejor, por su parte, no equivale ni si quiera a que probablemente sea el mejor curso de acción. Aun si se tiene la certeza de que un curso de acción no es el que posee el mayor valor actual, y por ende no es el objetivamente mejor, es posible que prospectivamente lo sea.

El valor esperado de un acto es una función de los valores actuales que tienen sus posibles resultados ponderados por la probabilidad de que acaezcan. La probabilidad relevante aquí es de índole epistémica. Este tipo de probabilidad tiene que ver con el grado de certeza con relación a ciertas proposiciones que está justificado que un agente posea en base a cierto cuerpo de evidencia. Explicando la probabilidad epistémica, señala Zimmerman: "Si una proposición, p, es cierta para alguien, S (esto es, si S está justificado epistémicamente en tener completa confianza en p), entonces la probabilidad de p para $S$ es 1. Si p es cierta para $S$, entonces su negación, - $p$, es ciertamente falsa para $S$; en este caso, la probabilidad de - p para $S$ es 0 . Si $p$ y - $p$ están contrabalanceadas para $S$ (esto es, $S$ está justificado en tener alguna confianza tanto en $p$ como en $-p$ ), entonces la probabilidad de cada una de estas proposiciones, $p$ y $-p$, para $S$ es 0.5. Si S está justificado en tener mayor confianza en $p$ que en $-p$, entonces la probabilidad de $p$ para $S$ es mayor que 0.5 y a probabilidad de $-p$ es menor que 0.5; en tal caso, $p$ puede decirse que es probable para $S, y-p$ improbable".${ }^{17}$ Sin embargo, el curso de acción prospectivamente mejor no es meramente aquel que tiene el mayor valor esperado. Esto porque el valor esperado de una opción es sensible solo a la cantidad de evidencia, pero es ciego al grado de confiabilidad que la misma posee. Por el contrario, lo prospectivamente mejor es una función no solo de la evidencia disponible, sino -adicionalmente- del grado de confiabilidad de la evidencia. Lo prospectivamente mejor es una función del valor esperado y de la confiabilidad de la evidencia. Poniendo juntas todas estas consideraciones puede señalarse que el curso de acción prospectivamente mejor es aquel que tiene el mayor valor esperado, donde lo relevante es la probabilidad epistémica determinada a partir de la evidencia disponible para el agente y donde la evidencia es ponderada según su grado de confiabilidad. ${ }^{18}$

A pesar de que en el texto tendré ocasión de introducir ulteriores refinamientos, he optado por una versión simplificada porque creo que la misma posee el grado de complejidad requerido para abordar el problema que aborda el presente trabajo.

17 Zimmerman (2008) p. 36.

18 Existen otros refinamientos que deben ser llevados adelante para que la concepción prospectiva pueda acomodar todas nuestras intuiciones morales. [...] Uno señala que aquello que debemos hacer no solo es una función de la evidencia disponible con relación a los 
SELEME, HUGO OMAR: PRESUNCIÓN DE INOCENCIA Y CONCEPCIONES DE LA OBLIGACIÓN MORAL

\section{Adoptar esta concepción prospectiva de obligación permite interpretar}

resultados posibles de nuestras acciones, sino también a la evidencia de la evidencia disponible con relación al valor actual de cada resultado. Para referirse a este tipo de valor que también es sensible al grado de probabilidad de que un resultado posea un valor actual, Zimmerman (2008) pp. 38 y 39 utiliza el rótulo de valor expectable. Distinguiendo el valor esperado del valor expectable, señala: "Mientras que el valor esperado, EV, de un acto es una función de las probabilidades de sus posibles resultados y de los valores actuales asociados con esos resultados, el valor espectable, $E^{*} \mathrm{~V}$, de un acto es una función de las probabilidades de sus posibles resultados y de los probables valores asociados con estos resultados [...]". Lo que la concepción prospectiva sostiene, una vez agregada esta precisión, es que "[u]n agente debe realizar un acto si y solo si es la opción que tiene el mayor valor espectable para el agente".

Otro refinamiento sostiene que lo que debemos hacer no solo es sensible a la evidencia disponible con relación a los posibles resultados de nuestras opciones y de los valores probables de esos resultados, sino adicionalmente a la evidencia de la que disponemos respecto de esta evidencia. El punto de Zimmerman (2008) pp. 39 y 40, es que el hecho de que alguien esté justificado en creer algo no implica que su creencia de que está justificado en creerlo lo esté. En su opinión, la evidencia respecto del valor expectable de una alternativa puede ser tan defectuosa como la evidencia respecto de su valor actual, y en este caso lo relevante es el valor expectable del valor expectable de la alternativa. El hecho de que existan diferentes niveles de evidencia necesariamente conlleva esta conclusión.

Como pueden existir distintos niveles de evidencia, esta conclusión amenaza un regreso al infinito. Sin embargo, dadas las limitaciones epistémicas de los seres humanos, agrega ZIMMERMAN (2008) p. 41, existirá un nivel L de evidencia más allá del cual no nos estará disponible otro o, de haberlo, será uno tal que el maximizar el valor expectable a ese nivel también lo hará al nivel L. En este caso, L es el nivel definitivo de evidencia para un agente. Con esta precisión en mente, ZimMerman (2008) p. 42 vuelve a modificar el enunciado de la concepción prospectiva: "Un agente debe realizar una acción si y solo si es la acción que tiene el mayor valor expectable para el agente a su nivel definitivo de evidencia".

Por último, dos consideraciones deben tenerse en cuenta a la hora de interpretar el valor prospectivo de una acción como equivalente a su valor esperado o expectable. En primer lugar, no siempre consideramos adecuado comportarnos como agentes que buscan maximizar el valor esperado. Zimmerman (2008) pp. 53 y 54 utiliza la paradoja de Allais para mostrar que la apelación al valor esperado debe ser cualificada de modo de dar cuenta de esta complejidad.

En segundo lugar, aunque dos alternativas tengan el mismo valor expectable -que se calcula a partir del valor esperado-, un agente puede estar justificado en tratarlas de modo distinto si una de ellas tiene para él un alto costo personal. Si queremos ver en estas alternativas personalmente costosas posibles actos supererogatorios, no es posible incluir el costo personal a la hora de determinar que estamos obligados a hacer. De modo que la concepción prospectiva debe refinarse para señalar que es obligatorio realizar la acción 
de una nueva manera la obligación estatal de castigar y absolver respetando la norma penal sustantiva. La norma penal sigue fijando cual es el mejor curso de acción, a saber, condenar a los transgresores y absolver a los inocentes. No obstante, al igual que sucede si uno adopta la concepción subjetiva de obligación, el Estado no tiene la obligación de seguir el mejor curso de acción. La obligación moral del Estado no es condenar al culpable y absolver al inocente. Su obligación moral es adoptar el curso de acción -condenar o absolver- que sea mejor prospectivamente dado el valor actual de condenar o absolver a personas inocentes o culpables, dada la probabilidad epistémica de que el imputado sea culpable o inocente medida a partir de la evidencia producida en el juicio y disponible para el Estado, y de la confiabilidad de la misma.

Mientras que si uno adopta una concepción objetivista de obligación lo relevante a la hora de fijar la obligación estatal de castigar o absolver es la verdad acerca de los hechos debatidos en el proceso, y si se adopta una concepción subjetivista son las creencias de quien tiene la potestad para fijar los hechos en el proceso, lo que es relevante si se toma una concepción prospectiva es la cantidad y calidad de la evidencia acerca de los hechos que está disponible en el proceso. Si el Estado administra el castigo solo a aqueIlos que dada la evidencia disponible es probable que hayan transgredido la norma, entonces ha cumplido su obligación moral.

Que el Estado ignorase cuál era la verdad acerca de los hechos a la hora de administrar el castigo no funciona como una excusa al reproche por haber transgredido su obligación moral de castigar al culpable y absolver al inocente. Tampoco altera los términos de la obligación estatal, tal como sostiene la concepción subjetivista de obligación. Lo que fija la obligación estatal no son los hechos -como sostiene la concepción objetivista- ni las creencias del juzgador -como sostiene la subjetivista-, sino la evidencia disponible. Si el juzgador absuelve a una persona que cree que es culpable, y en realidad lo es, debido a que la evidencia inculpatoria disponible en el proceso no es suficiente, entonces el Estado ha cumplido con su obligación moral. Por el contrario, si condena a quien cree culpable, y en realidad lo es, pero lo hace

cuyo resultado tiene el más alto valor expectable, siempre y cuando no requiera un alto costo personal. Cfr. ZIMMERMAN (2008) pp. 55 y 56.

Existen otros refinamientos que no he consignado porque creo que con lo señalado el lector puede tener una visión suficientemente precisa de lo que sostiene la concepción prospectiva. A los fines del argumento que presento en el texto debe entenderse que las circunstancias que dan lugar a los refinamientos que he reseñado, no se encuentran configuradas. 
sobre la base de evidencia insuficiente, entonces el Estado no ha cumplido su obligación moral a la hora de distribuir el castigo.

\section{CONCEPCIONES DE OBLIGACIÓN Y PRINCIPIO DE INOCENCIA}

Cada una de estos modos de concebir la obligación estatal de castigar y absolver respetando lo establecido por la norma penal sustantiva trae aparejado una manera diferente de concebir el Principio de Inocencia. Lo único que es necesario agregar es una premisa compartida por todos los juristas, que sostiene que la actividad probatoria se encuentra sometida a exigencias legales y que el Estado debe fundar su juicio sobre los elementos que obran en el proceso judicial. Prueba de la centralidad que esta idea posee es que el principio "quod non est in actis non est in mundo" remonta sus orígenes hasta el Derecho Romano, aun si se consolidó como un principio jurídico en el siglo XIII. ${ }^{19}$ Una consecuencia de este principio es que el decisor no puede fundar su juicio en elementos que se encentren fuera del proceso o que hayan sido incluidos vulnerando las exigencias legales. De acuerdo con Damaska: "la opinión prevaleciente era que el conocimiento privado y el oficial no debían ser mezclados, y que la toma de decisión debía estar fundada únicamente sobre la información adquirida de manera oficial". ${ }^{20}$

Si uno combina la premisa de que el decisor debe fundar su juicio en los elementos obrantes en el proceso con una concepción objetivista de la obligación estatal, es plausible entender que el principio se refiere a la inocencia material del acusado. Si solo los elementos probatorios incorporados al proceso son relevantes y, adicionalmente, el Estado solo debe castigar a los culpables, es natural concluir que lo que determina la culpabilidad de un individuo son los elementos que obran en el proceso judicial.

Esto conduce a interpretar al principio de inocencia como estipulando que toda persona es inocente, esto es no ha transgredido la norma penal sustantiva, hasta tanto haya sido establecido lo contrario en el proceso judicial. No se trata meramente de que deba ser tratado "como si fuese" inocente antes de que haya sido determinada su culpabilidad, sino de que hasta tanto esto suceda "es" inocente y por eso debe ser tratado de esa manera. Larry Laudan reconstruye este modo de interpretar al Principio de Inocencia como la: "tendencia novedosa pero actualmente de moda de suponer que si alguien

\footnotetext{
19 Una de las primeras recepciones del principio se encuentra en la decretal de 1216 del Papa Inocencio III. Aunque el principio estuvo en un primer momento unido al carácter escrito del proceso judicial, su aplicación ha sido extendida a los procesos orales.

20 DAMASKA (1986) p. 30.
} 
es culpable o inocente de un crimen depende de que la prueba ofrecida en el juicio sea suficiente para persuadir a una persona racional de que el acusado es culpable". ${ }^{21}$

Algunas expresiones en este sentido suenan llamativas por la peculiar teoría de la verdad sobre la que aparentemente descansan. Así, Henry Chambers, haciendo patente este compromiso con una concepción peculiar de la verdad, ha señalado: "En el proceso judicial, la inocencia o la culpabilidad real del acusado puede ser una abstracción. Lo que es verdad es aquello que la prueba indica que es verdad". ${ }^{22}$

Si uno acepta las dos premisas -referidas a la concepción objetiva de obligación y a la exigencia de fundar el juicio en los elementos incorporados al proceso- y desea evitar la conclusión de que la evidencia incorporada al proceso es la que determina la verdad, la única alternativa disponible es señalar que en ciertas ocasiones cuando el Estado actúa sobre la base de la evidencia disponible incumple su obligación de castigar al culpable y absolver al inocente. Tal situación se presenta cuando la evidencia es defectuosa. A pesar de que aparentemente el Estado no ha hecho nada que sea incorrecto -dado que se ha ajustado a la evidencia disponible-, debe concluirse que se ha comportado de manera inadecuada. El carácter defectuoso de la evidencia actúa simplemente como una excusa para el reproche moral que genera la obligación estatal incumplida.

De modo que quien acepta las dos premisas se ve conducido a un dilema cuyos cuernos son igualmente problemáticos. Dado que el Estado ha actuado sobre la base de la evidencia disponible no existe ningún reproche que se le pueda dirigir. Si movido por esta convicción concluye que el Estado ha cumplido con su obligación -aun en el caso de que la evidencia de pie a un juicio falso- y posee una concepción objetivista de obligación, entonces debe necesariamente asumir que lo que es verdad es aquello que la evidencia determina como verdad. El proceso constituye aquello que es verdad. Si, por el contrario, concluye que el Estado no es reprochable meramente porque la evidencia defectuosa actúa como una excusa, entonces debe asumir que aunque el Estado ha seguido todas las pautas procesales, no ha cumplido con su obligación moral de castigar y absolver.

El carácter altamente problemático que tiene este segundo extremo que implica asumir que el Estado, aunque cuenta con reglas procesales

\footnotetext{
21 LAUDAN (2006) p. 11.

22 Chambers (1998) p. 668.
} 
completamente justificadas y se ha comportado de modo diligente en la recolección y evaluación de las pruebas, es posible que haya incumplido con su obligación moral de castigar y absolver- explica en parte por qué, como señala Laudan, la concepción de la verdad constituida por la evidencia disponible ha cobrado auge en el último tiempo.

Si se combina la premisa que manda fundar el juicio en los elementos probatorios incorporados al proceso con una concepción subjetivista de la obligación estatal de castigar, aparece una manera alternativa de concebir el Principio de Inocencia. Dado que para este modo de concebir la obligación moral lo relevante son las creencias del agente, el principio ahora debe ser entendido como prescribiendo qué creencias debe tener el encargado de juzgar los hechos. Específicamente, el principio establece que el juzgador a la hora de iniciarse el proceso debe creer en la inocencia del acusado. El principio pretende garantizar que las creencias del juzgador hayan sido generadas solo por los elementos probatorios obrantes en la causa.

Este modo subjetivo de concebir la obligación estatal de castigar permite explicar por qué ha sido usual utilizar estándares de prueba subjetivos en el proceso judicial. La diferentes interpretaciones que se han ofrecido del estándar de "duda razonable" no son sino solo un ejemplo. En sus orígenes el estándar de prueba judicial exigía que el juzgador tuviese completa certeza de la culpabilidad del acusado antes de dictar un veredicto condenatorio. Por supuesto, esta certeza debía provenir de los elementos probatorios incorporados al proceso y el juzgador debía partir de la creencia de que el acusado era inocente. Tanto el principio de inocencia como el estándar de prueba tenían un carácter subjetivo.

Este carácter subjetivo permaneció inalterado cuando se pasó del estándar de la certeza al de la "duda razonable". Tampoco ha perdido su carácter subjetivo el modo correlativo de interpretar el principio de inocencia. Ante la imposibilidad demostrada de que pudiese alcanzarse una completa certeza sobre asuntos contingentes como los abordados en un proceso judicial, el estándar de prueba se volvió menos exigente. Ahora no se exigía certeza sino solo certeza moral. ${ }^{23}$ De acuerdo con Laudan: "Lo que caracterizaba a las creencias moralmente ciertas era que, aunque en teoría se encontraban

23 La formulación canónica del principio de "duda razonable" en términos de "certeza moral" se encuentra en el fallo dictado en la causa Commonwealth v. Webster por la Corte Suprema del Estado de Massachusetts durante el año 1850. El juez Lemuel Shaw, presidiendo la Corte, señaló en su voto que "the evidence must establish the truth of the fact to a reasonable and moral certainty". 
sujetas a la duda de los escépticos, no existía en la práctica ningún fundamento real o racional para dudar. De aquí surgió la idea de que un veredicto de culpabilidad exigía que el jurado creyese más allá de una duda razonable o con certeza moral en la culpabilidad del acusado". ${ }^{24}$

Por razones vinculadas con el carácter problemático de apelar a cualquier estándar moral, la interpretación de la "duda razonable" a partir de la "certeza moral" fue abandonada. A la vez que la Corte Suprema estadounidense adoptaba ese estándar de prueba, señalaba que el mismo no podía definirse a partir de la certeza moral ${ }^{25}$, porque esto podía transmitir al juzgador la errónea idea de que podía basar sus creencias acerca de la culpabilidad del acusado en sentimientos morales o emociones, en lugar de hacerlo sobre la evidencia. ${ }^{26}$ Desde entonces diferentes maneras de interpretar la "duda razonable" han sido ofrecidas por la dogmática y los tribunales estadounidenses. ${ }^{27}$

Todas estas interpretaciones alternativas han mantenido su carácter subjetivo. Según la primera, lo que la "duda razonable" exige es que a la hora de condenar el juzgador crea que el acusado es culpable con la misma fuerza que tienen las creencias sobre las que basa decisiones vitales importantes. El principio de inocencia le exige creer al juzgador que el acusado no es culpable y, correlativamente, el estándar de la "duda razonable" solo corporiza la idea de que esta creencia en la inocencia solo puede ser derrotada por otra en sentido contrario dotada con la misma fuerza que poseen las convicciones

$24 \quad$ LAUDAN (2006) p. 33.

25 En el fallo Victor v. Nebraska de 1994 la jueza de la Corte Suprema de Estados Unidos, Sandra Day O'Connor, señalaba de modo contundente que "(...) this Court does not condone the use of the antiquated 'moral certainty' phrase (...)". No obstante, la jueza consideraba que el uso de esta expresión en las instrucciones brindadas al jurado no volvía inconstitucional la sentencia bajo revisión. En el mismo fallo el juez Harry Blackmun consideró que la referencia a la certeza moral en las instrucciones brindadas al jurado vulneraba la cláusula del Debido Proceso de la Constitución de Estados Unidos. Tal utilización, señalaba Blackmun, ya había sido desacreditada por la Corte en 1990 en el caso Cage donde se establece que "(...) the reference to 'moral certainty', rather than evidentiary certainty, it becomes clear that a reasonable juror could have interpreted the instruction to allow a finding of guilt based on a degree of proof below that required by the Due Process Clause".

26 El estándar de la "duda razonable" fue dotado de rango constitucional por la Corte Suprema estadounidense en el caso Winship de 1970. La Corte señaló que el estándar debía entenderse que se encontraba incorporado en la Decimocuarta Enmienda.

27 Para presentar las diferentes maneras en las que ha sido concebida la "duda razonable" he seguido lo que señala LAUDAN (2006), p. 36 y ss. 
sobre las que hemos adoptado las decisiones que poseen mayor relevancia en nuestra propia vida. ${ }^{28}$

Según la segunda interpretación, el estándar de la "duda razonable" requiere que el juzgador condene solo si cree en la culpabilidad del acusado, y lo hace con un grado de certeza tal que haría que una persona prudente no dudase en actuar. Si una persona prudente no tendría dudas en condenar al acusado, de tener la misma creencia en su culpabilidad que posee el juzgador, entonces el estándar de la "duda razonable" ha sido satisfecho. ${ }^{29}$

La tercera interpretación también señala que el juzgador debe condenar si cree que el acusado es culpable. Solo que ahora debe tratarse de una creencia arraigada, estable. Solo si el juzgador tiene una creencia firme -como opuesta a cambiante- en la culpabilidad de aquel a quien se acusa, está justificado que ordene su condena. ${ }^{30}$

Por último, la cuarta interpretación también posee carácter subjetivo. De acuerdo con este modo de concebir la "duda razonable", el juzgador debe condenar solo si no existe ninguna duda sobre la culpabilidad del acusado para la que pueda ofrecerse alguna razón. Dicho de otro modo, una duda es razonable si puede brindarse algún tipo de razón que la respalde. Por el contrario, si la duda es infundada, entonces es irrazonable. ${ }^{31}$

28 Una muestra de la utilización de este estándar puede encontrarse, señala Laudan, en el modelo de instrucción para los jurados emitido por la Corte del Quinto Circuito, que señala en su punto 1.5: "A 'reasonable doubt' is a doubt based upon reason and common sense after careful and impartial consideration of all the evidence in the case. Proof beyond a reasonable doubt, therefore, is proof of such a convincing character that you would be willing to rely and act upon it without hesitation in making the most important decisions of your own affairs".

29 Esta interpretación del estándar ha sido incorporada en el modelo de instrucción para los jurados elaborado por la Corte del Segundo Circuito. Allí se afirma que "A reasonable doubt is a doubt based on reason and common sense - the kind of doubt that would make a reasonable person hesitate to act. Proof beyond a reasonable doubt must, therefore, be proof of such a convincing character that a reasonable person would not hesitate to rely and act upon it in the most important of his own affairs".

30 La interpretación ha sido adoptada por la instrucción n 103 del Judicial Council of California que sostiene: "Proof beyond a reasonable doubt is proof that leaves you with an abiding conviction that the charge is true. The evidence need not eliminate all possible doubt because everything in life is open to some possible or imaginary doubt".

31 Esta interpretación fue adoptada por diferentes tribunales y declarada errónea por sendos fallos de los tribunales de alzada. Así, por ejemplo, el tribunal de juicio la adoptó en el 
El hecho de que el estándar de prueba judicial sea concebido en términos subjetivos ha comenzado recientemente a preocupar a los juristas. De acuerdo con Laudan: "Esta focalización en el estado mental del jurado es algo más que una pequeña curiosidad. Supongamos que tratamos de enseñar a jóvenes científicos cuándo una teoría fue aceptable diciéndoles cuál debería ser su estado mental cuando ellos deberían aceptar una teoría. Tal consejo sería visto como una tontería. Trate de imaginar a un matemático señalando que ha probado un nuevo teorema y que la prueba consiste en el hecho de que él cree el teorema sin la menor duda. Sus colegas estarían sorprendidos puesto que lo que establece que un teorema matemático es un teorema es el carácter robusto de su prueba, no la confianza de su descubridor". ${ }^{32}$

Esta curiosidad propia del proceso judicial -consistente en adoptar un estándar subjetivo de prueba- puede ser explicada a partir de la adopción de una concepción subjetiva de obligación. Si uno adopta una concepción subjetiva de la obligación moral, e interpreta a partir de ella la obligación estatal de castigar respetando lo establecido por la norma penal sustantiva, es natural focalizarse en los estados mentales del juzgador. Siendo este el caso, es igualmente natural interpretar al principio de inocencia como prescribiendo cuáles son las creencias que debe tener el encargado de determinar los hechos al momento de iniciarse el proceso.

Finalmente, si se adopta una concepción prospectiva de obligación moral y se la combina con el principio que sostiene que lo único que posee relevancia son los elementos probatorios incorporados al proceso, aparece un tercer modo de concebir el Principio de Inocencia. Puesto que para la concepción prospectiva de obligación lo que interesa es solo la evidencia disponible para el Estado -esto es la efectivamente incorporada al proceso-, el principio simplemente prescribe al encargado de juzgar los hechos que acepte al iniciarse el proceso que no existe ninguna evidencia en contra del acusado. El principio de inocencia simplemente le recuerda al juzgador que cuando el proceso se inicia no existe ninguna evidencia disponible para el Estado. El hecho de que el acusado haya sido considerado como sospechoso de cometer un delito, haya sido detenido y sometido a juicio, no deben constituir evidencia de su culpabilidad para quien va a juzgar los hechos.

Laudan propone interpretar el principio de inocencia de esta última manera, aunque no vincula su propuesta con un modo específico de concebir la

fallo Burnett v. Nebraska de 1910. La Corte de Apelación sostuvo que se trataba de un error y revirtió la sentencia.

32 Laudan (2006) pp. 51 y 52. 
obligación estatal de castigar. En primer lugar, propone distinguir entre lo que denomina "inocencia material" e "inocencia probatoria". La primera hace referencia al hecho de que el acusado no ha transgredido efectivamente el mandato legal. La segunda, en cambio, solo señala que no existe evidencia disponible para el Estado que permita concluir que la transgresión ha sido cometida. ${ }^{33}$ En segundo lugar, sostiene que el Principio de Inocencia se vincula con la "inocencia probatoria".

Siguiendo esta línea de pensamiento, Laudan afirma que un buen evaluador de los hechos "es alguien dotado de apertura mental acerca del resultado eventual del proceso judicial y no se siente bajo ninguna obligación, ni siquiera en una posición, de asignar probabilidades -empatadas o no- acerca del resultado del proceso. Lo que es importante es que el jurado reconozca que ahora no tiene ninguna prueba sobre la culpabilidad y que, por lo tanto, carece de cualquier indicio sobre qué lado eventualmente prevalecerá. El punto crucial es que el jurado debe estar dispuesto a aceptar, sin ninguna reserva, la tesis de la inocencia, sin importar las hipótesis tentativas que pueda haber tenido sobre la culpabilidad y la inocencia". ${ }^{34}$

Resumiendo lo expuesto hasta aquí, existen tres maneras alternativas de concebir el Principio de Inocencia según que se adopte una concepción objetiva, subjetiva o prospectiva de la obligación estatal de castigar. En el primer supuesto, el principio señala que toda persona "es" inocente si no ha sido demostrado en juicio lo contrario. En el segundo, el principio le indica al juzgador que al momento de iniciarse el proceso debe creer en la inocencia material del acusado. Finalmente, si uno se inclina por la tercera alternativa el principio le prescribe al juzgador que acepte que al iniciarse el proceso no existe ninguna evidencia a favor de la culpabilidad del acusado.

33 De acuerdo con LaUdAN (2006) p. 12, "es legítimo, y en algunos contextos esencial, distinguir entre la aseveración que \%ones is culpable" en el sentido que ha cometido un crimen, y la aseveración que "Jones es culpable" en el sentido que el sistema legal lo ha condenado. Propongo denominar al primer sentido culpa material (en lo sucesivo, culpam) y al segundo culpa probatoria (culpap). Claramente, la culpap no implica la culpam y viceversa. De manera similar, podemos distinguir entre la inocencia material de Jones (inocenciam), en el sentido que él no cometió el crimen, y su inocencia probatoria (inocenciap), en el sentido que fue absuelto o de algún otro modo se vio liberado del escrutinio judicial".

34 LAUDAN (2006) pp. 105 y 106. 


\section{DE LA CONCEPCIÓN CORRECTA DE OBLIGACIÓN A LA CONCEP- CIÓN CORRECTA DEL PRINCIPIO DE INOCENCIA}

Las razones que se han ofrecido en la literatura para inclinarse por alguna interpretación del principio de inocencia han sido variadas. Mientras algunos, como Laudan, han apelado a las funciones que el principio desempeña dentro del proceso judicial para mostrar que concebirlo de cierto modo es inapropiado e innecesario ${ }^{35}$; otros, como Antony Duff ${ }^{36}$, han elaborado su interpretación preferida a partir de consideraciones de moralidad política. ${ }^{37}$

Las que voy a ofrecer en esta sección son razones de un tipo diferente. Habiendo establecido que las interpretaciones del principio de inocencia se apoyan en diferentes maneras de concebir la obligación moral, me dedicaré a mostrar cuál de estas concepciones considero que es la correcta. Específicamente, enunciaré las razones que pueden ofrecerse a favor de una concepción prospectiva de obligación. El resultado final de este movimiento argumentativo habrá sido construir un caso a favor de la interpretación del Principio de Inocencia según la cual éste simplemente prescribe al juzgador aceptar que al inicio del proceso no existe ninguna evidencia que respalde la culpabilidad del acusado.

Zimmerman ha dirigido un poderoso ataque en contra de las concepciones objetiva y subjetiva de obligación, argumentando a la vez a favor de la variante prospectiva. Por lo que respecta a la concepción subjetiva Zimmerman ha señalado algunas de las consecuencias contraintuitivas que se siguen de adoptarla. En primer lugar, si la concepción fuese cierta entonces todos los agentes poseerían una especie de infalibilidad moral. Dado que la concepción vuelve nuestras obligaciones morales una función de nuestras creencias, y dado que tenemos un acceso directo a nuestras propias creencias, determinar a qué estamos obligados sería solo una cuestión de introspección. Así como no podemos equivocarnos a la hora de determinar lo que creemos, si excluimos los errores de inferencia, tampoco podríamos equivocarnos al determinar a qué estamos moralmente obligados. ${ }^{38}$

\footnotetext{
35 LAUDAN (2006) pp. 100 y ss.

36 Duff (2013).

37 También he ofrecido una interpretación del principio de inocencia -semejante a la que defiendo en este texto- sobre la base de consideraciones de moralidad política en "Legitimidad, Lealtad Cívica e Inocencia", Revista Jurídica de la Universidad San Andrés: paper en prensa.

$38 \quad$ ZimMERMAN (2008) pp. 13 y 14.
} 

CIÓN MORAL

En segundo lugar, de ser cierta la concepción subjetiva de obligación, existiría un modo expeditivo y espurio de eximirnos de nuestras obligaciones morales. Bastaría simplemente que no prestásemos la suficiente atención a las circunstancias en las que nos encontramos. Si debido a nuestra propia negligencia fallamos en advertir que un curso de acción es el mejor, y por tanto no llegamos a creer que lo es, entonces no estamos moralmente obligados a realizarlo. ${ }^{39}$ En tercer término, bastaría que alguien creyese que lo que está llevando adelante es el mejor curso de acción, para que haya cumplido su obligación moral. Sin importar la cantidad y calidad de la evidencia sobre la que funda su creencia, actuar de acuerdo con ella sería suficiente para satisfacer su obligación. Así, por ejemplo, sería suficiente que Hitler hubiese creído que matar judíos era el mejor curso de acción, para que nos viésemos conducidos a sostener que se encontraba moralmente obligado a hacerlo. ${ }^{40}$

Por último, Zimmerman arguye que aceptar la concepción subjetiva de obligación moral conduce a vulnerar el principio de que "debe" implica "puede". Si creemos falsamente que podemos hacer algo y adicionalmente creemos que se trata del mejor curso de acción, entonces con total independencia de que en realidad no sea posible para nosotros realizar la acción, tenemos la obligación moral de realizarla. ${ }^{41}$

Las razones en contra de la concepción objetivista de obligación son más sutiles. Algunos casos hipotéticos, ideados por el propio Zimmerman, pueden ser de utilidad para comprenderlas. Imaginemos que una médica (Jill) tiene un paciente (John) quien sufre de una afección a la piel. Jill tiene la opción de administrarle a John tres drogas: A, B y C. Jill, adicionalmente, dispone de cierta evidencia sobre los resultados que serán producidos por cada droga: "Toda la evidencia de la que dispone Jill indica (de modo acertado) que darle a John la droga B lo curaría parcialmente y darle ninguna droga volvería su enfermedad incurable, pero también indica (de modo desacertado) que darle la droga C lo curaría completamente y darle la droga A lo mataría". ${ }^{42}$ En realidad, en contra de la evidencia disponible, darle la droga $C$ lo matará y darle la A lo curará completamente.

Frente a este caso, quienes defienden la concepción objetivista de obligación, señalarían que, si la médica, basada en la evidencia, le da la droga C -causándole la muerte- no ha cumplido su obligación moral, puesto que

\footnotetext{
39 Ídem., p. 14.

40 Ídem., p. 15.

41 ldem., pp. 15 y 16.

42 Ídem., p. 17.
} 
el mejor curso de acción -contrario a lo que la evidencia sugería- era administrarle la droga A. No obstante, su falta de conocimiento hace que la transgresión no le sea moralmente reprochable. Este modo de interpretar el caso le permite a los objetivistas acomodar la intuición moral de que nada puede reprocharse al médico, sin tener que concluir que nada tenemos para reprocharle porque no hay ninguna obligación que haya transgredido. Su obligación era adoptar el mejor curso de acción, señala el objetivista, y no la ha cumplido. No obstante, no hay reproche porque la errónea evidencia de la que disponía actúa como excusa moral.

Trasladado a la obligación estatal de castigar, si el Estado no condena a un culpable debido a la falta de evidencia, entonces no es reprochable moralmente por haber incumplido con su obligación. Esta manera de entender la obligación de castigar da lugar a una curiosa asimetría que he explorado en otro lugar. ${ }^{43}$ Aunque el Estado puede excusarse en base a la falta de evidencia, el mismo camino no queda disponible para el abogado que, conociendo la culpabilidad de su cliente, arguye por su absolución cuestionando la validez y la fuerza del material probatorio. Tal cosa es así porque la evidencia disponible para el Estado es diferente de la que está disponible para el abogado que conoce la culpabilidad de su defendido. El abogado no puede esgrimir ignorancia para eximirse del reproche moral por incumplir el deber de no contribuir de modo deliberado a que el Estado no satisfaga su obligación de castigar al culpable. ${ }^{44}$

La clave de la respuesta objetivista al caso recién planteado es, por un lado, la distinción entre que un acto sea moralmente incorrecto y que el agente que lo realiza sea moralmente reprochable y, por otro, la tesis de que la incertidumbre o la evidencia defectuosa es una excusa a la reprochabilidad pero no incide a la hora de determinar cuál es la acción correcta. Lo correcto sigue siendo elegir la alternativa cuyos resultados son efectivamente los mejores.

No obstante, si uno altera ligeramente el caso, la respuesta de la concepción objetiva pierde su plausibilidad, lo cual es una razón para cuestionar su corrección. El caso que sirve para cuestionar a la concepción objetiva es uno

Seleme (2013).

44 Aunque creo que esta asimetría entre el reproche moral que recibe el Estado y el abogado puede verse como un indicio de lo defectuoso de la concepción objetiva de obligación, no perseguiré este punto aquí. 

CIÓN MORAL

ideado por Frank Jackson. ${ }^{45}$ El mismo es semejante al recién presentado, solo cambia la evidencia disponible. Ahora, "toda la evidencia de la que dispone Jill indica (de modo acertado) que darle a John la droga B lo curaría parcialmente y no darle ninguna droga volvería su enfermedad incurable, pero deja completamente indeterminado si darle la droga A o la C lo curaría completamente o si darle la droga A o la C lo mataría". ${ }^{46}$ En estas circunstancias, ¿qué debería hacer Jill?, ¿cuál es su obligación moral?

Nuestra intuición moral frente a este caso es que la médica tiene la obligación moral de administrarle al paciente la droga B. No obstante, esta es una respuesta de la cual la concepción objetiva no puede dar cuenta. La distinción entre la incorrección de la acción y la reprochabilidad moral del agente -y la apelación a la incertidumbre como excusa del reproche moral- no son aquí de utilidad. Jill no puede decir -como sí podía en el caso anterior-que al darle la droga B estaba intentando hacer lo que era mejor para John dada la evidencia de la que disponía. La razón de ello es clara, en este caso la evidencia indica que darle la droga B no producirá el mejor resultado. El mejor resultado se produciría si le diese la droga $\mathrm{A}$ o $\mathrm{C}$. Administrarle la droga $\mathrm{B}$ es el segundo mejor curso de acción, no el primero. Que nuestra intuición frente al caso sea que jill está obligada moralmente a administrar la droga B muestra que no consideramos que lo moralmente obligatorio sea adoptar el mejor curso de acción disponible para el agente. Es decir, no consideramos que la concepción objetiva de obligación sea correcta.

El ejemplo adicionalmente sirve para mostrar que la concepción de obligación que permite acomodar nuestras intuiciones es la prospectiva. De acuerdo con Zimmerman, darle a John la droga B es lo que llamaré prospectivamente mejor, en tanto es lo que le da a Jill la mejor perspectiva de alcanzar lo que es de valor en la situación (a saber, la recuperación de la salud de John). ${ }^{47}$ Como he señalado, y el caso muestra con claridad, que algo sea prospectivamente mejor, por su parte, no equivale a que probablemente sea lo mejor. En el caso de Jackson, Jill sabe con total certeza que darle la droga B no es el mejor curso de acción, sino el segundo mejor, y aun así cree que es lo mejor que puede hacer. Es lo mejor en sentido prospectivo. Lo prospectivamente mejor es el curso de acción que maximiza el valor esperado.

45 Zimmerman (2008), pp. 9 y 10, reconoce que este caso fue el que le hizo revisar su convicción de que la incertidumbre debía funcionar solo como una excusa al reproche moral pero no debía alterar nuestras obligaciones morales.

46 La presentación que hace ZIMMERMAN del caso es ligeramente diferente de la que hace Jackson, aunque ambas versiones son idénticas en sus elementos esenciales.

47 Zimmerman (2008) pp. 18 y 19. 
Para entender la idea puede ser de utilidad ofrecer la reconstrucción formal que Zimmerman hace del caso de Jill utilizando la idea de valores esperados en base a probabilidades epistémicas. Dado que existen cuatro posibles resultados -cura total, cura parcial, incurabilidad y muerte- y que el mejor resultado es el primero y el peor el último, imaginemos que sus valores actuales son respectivamente 50, 40, 0 y -100. Las opciones, o cursos de acción alternativos son cuatro: administrar la droga $A$, la B, la $C$ o no administrarle ninguna. La probabilidad -en base a la evidencia disponible para Jill- de que cada curso de acción produzca cierto resultado son: si le administra la droga B existe una certeza completa de cura parcial (la probabilidad de cura parcial es 1), si le administra la A existe una igual probabilidad de cura total o muerte (la probabilidad de cura total es 0,5 y la probabilidad de muerte es 0,5 ), idénticas probabilidades se aplican a la opción de administrarle la droga C, finalmente, si no le administra ninguna droga existe una certeza completa de incurabilidad permanente (la probabilidad de incurabilidad es 1).

Si ponderamos el valor de cada resultado posible de un curso de acción con la probabilidad de que acaezca, obtenemos el valor esperado de dicho curso de acción. Así, el valor esperado de cada curso de acción es:

- Administrar $\mathrm{A}=[(50 \times 0,5)+(-100 \times 0,5)]=-25$

- Administrar $C=[(50 \times 0,5)+(-100 \times 0,5)]=-25$.

- Administrar $\mathrm{B}=(40 \times 1)=40$.

- No administrar ninguna $=(0 \times 1)=0^{48}$.

La intuición moral de que lo moralmente obligatorio es administrarle a John la droga $B$ muestra que no consideramos que sea obligatorio moralmente adoptar el mejor curso de acción, puesto que administrar la droga $B$ no es el curso de acción con el mayor valor actual. No obstante, administrar la droga B maximiza un valor, maximiza el valor esperado de acuerdo a la evidencia disponible para el agente, lo que sirve para dar sustento a la concepción prospectiva. ${ }^{49}$

48 Los valores y las probabilidades son atribuidos de modo arbitrario -puesto que se trata de un caso hipotético- pero a pesar de ello sirven para mostrar el atractivo de la concepción prospectiva.

49 La concepción prospectiva se diferencia tanto de la concepción objetiva -porque lo moralmente obligatorio es sensible al cuerpo de evidencia disponible para el sujeto- como de la subjetiva -porque lo moralmente obligatorio no depende de las creencias que el su- 

CIÓN MORAL

De modo que lo que muestra el caso es que nuestras obligaciones morales pueden verse alteradas de acuerdo a la evidencia que tengamos disponible en cada circunstancia. La obligación moral consiste en adoptar el curso de acción que es prospectivamente mejor, esto es, el que tiene el mayor valor esperado. El valor esperado de un curso de acción, por su parte, depende de la probabilidad epistémica de sus resultados, y el grado de probabilidad epistémica depende de la evidencia disponible para el agente.

Ahora bien, como he señalado al presentar la concepción prospectiva de obligación, lo prospectivamente mejor es una función no solo de la evidencia disponible, sino -adicionalmente- del grado de confiabilidad de la evidencia. Lo prospectivamente mejor es una función del valor esperado y de la confiabilidad de la evidencia. La idea puede también graficarse con una variante del caso de Jill. Imaginemos la siguiente situación: "Jill tiene que elegir entre dos drogas, $A$ y $B$, cual administrarle a John. Para cada droga la probabilidad para Jill de que administrarla cure completamente a John es 0,7, y la probabilidad de que no sea efectiva pero inocua es de 0.3. La droga $A$ ha sido ampliamente investigada; los datos son abundantes. La droga $B$ casi no ha sido investigada, en verdad los datos son muy pobres" ${ }^{50}$ En este caso, concluye Zimmerman, nuestra intuición moral es que Jill debería dar a John la droga A y no debería darle la B, aun si el valor esperado de cada opción es el mismo. Nuestra intuición sería la misma aun si el valor esperado de administrarle la droga A fuese ligeramente inferior al de darle B.

Otro caso hipotético puede servir de ayuda para trasladar lo señalado al dominio del proceso judicial. Imaginemos que un individuo se encuentra acusado de homicidio y la prueba rendida en el juicio ha sido meramente circunstancial. Sin embargo, el individuo es efectivamente culpable. El Estado -representado en aquel a quien ha conferido poder para juzgar los hechostiene dos cursos de acción alternativos: condenar al imputado o absolverlo. Cada uno de estos cursos de acción tiene dos resultados posibles: la condena

jeto efectivamente posea-. Si alguien quiere poner de manifiesto que la concepción prospectiva no es subjetiva diciendo que se trata de una concepción objetiva, no existe ningún problema en hacerlo. Lo único que es necesario tener en mente es que siguen existiendo tres tipos diferentes de concepciones de obligación: uno que es insensible a los estados mentales y a la evidencia disponible, otro que es sensible a la evidencia disponible y otro que es sensible a los estados mentales. Qué rótulo pongamos a estas concepciones -por ejemplo, que denominemos a las dos primeras como variantes de objetivismo que se diferencian de la tercera- no tiene ninguna consecuencia para el argumento que se ofrece en el texto. Agradezco a un árbitro anónimo el haberme sugerido explicitar este punto. 
de un culpable o la condena de un inocente; la absolución de un culpable o la absolución de un inocente. Dada la escasa evidencia confiable disponible para el decisor, supongamos que la probabilidad epistémica de que absuelva a un inocente es 0.9 y la de absolver a un culpable es 0.1 , la de condenar a un inocente es 0.9 y la de condenar a un culpable es 0.1.1. Imaginemos que los valores actuales de cada resultado reflejan que el mejor resultado posible es absolver a una persona si es inocente y el peor es condenarla. Con esto en mente los valores actuales podrían estipularse del siguiente modo: absolver a una persona inocente 100, condenar al culpable 80, absolver al culpable -80, y condenar al inocente -100. En el presente caso, dado que el imputado es culpable, condenar es el curso de acción que posee el mayor valor actual.

Si la concepción objetiva de obligación fuese correcta, entonces el Estado tendría la obligación de condenar, puesto que es el mejor curso de acción. Sin embargo, como he mostrado, la concepción objetiva es incorrecta y lo que el Estado debe hacer depende no solo del valor actual de cada curso de acción, sino de la probabilidad epistémica de cada uno de sus resultados posibles y de la confiabilidad de le evidencia sobre la que se funda. El Estado debe seguir el curso de acción que es prospectivamente mejor. El valor prospectivo de cada curso de acción para el caso es el siguiente:

Condenar $=(0.1$ probabilidad de culpa X 80$)+(0.9$ probabilidad de inocencia $X-100)=-82$.

Absolver $=(0.1$ probabilidad de culpa $X-80)+(0.9$ probabilidad de inocencia $X 100)=82$.

A pesar de que condenar es el mejor curso de acción tiene el mayor valor actual de las alternativas disponibles, 80 frente a -80 de absolver, absolver es el curso de acción prospectivamente mejor, con un valor de 82. Lo que el Estado está obligado a hacer en este caso es absolver, con total independencia de que el individuo efectivamente sea culpable. Su obligación moral es adoptar el curso de acción -condenar o absolver- que sea el mejor prospectivamente dado el valor actual de condenar o absolver a personas inocentes o culpables, dada la probabilidad epistémica de que el imputado sea culpable o inocente medida a partir de la evidencia producida en el juicio y disponible para el Estado y de la confiabilidad de la misma.

Si este es el modo correcto de concebir la obligación estatal de castigar, entonces el principio de inocencia no debe interpretarse ni como estipulando que toda persona "es" inocente si no ha sido demostrado en juicio lo contrario, ni como prescribiendo al juzgador que crea en la inocencia material del acusado al momento de iniciarse el proceso. Por el contrario, el principio 
manda al juzgador que acepte que al iniciarse el proceso no existe ninguna evidencia que sirva para inculpar al acusado. El principio meramente estipula que cualquier evidencia que el juzgador pudiese tener acerca de la culpabilidad o inocencia del acusado que no haya sido incorporada al proceso, carece de valor y no debe ser considerada a la hora de administrar el castigo.

\section{CONCLUSIONES}

El argumento que he presentado nos ha dejado con conclusiones de diferente nivel. En primer lugar, ha sido establecido que existe una relación entre el modo de concebir la obligación moral, la manera de entender la obligación estatal de castigar y absolver, y la forma en que se da contenido al principio de inocencia. Esta primera conclusión posee relevancia ya que las maneras corrientes de interpretar el Principio de Inocencia se apoyan en consideraciones vinculadas con el proceso judicial o con la teoría política y ninguna atención ha sido prestada a las consideraciones vinculadas con la manera en que se concibe la obligación moral.

En segundo lugar, han sido esquematizadas tres maneras diferentes de concebir al Principio de Inocencia según que la obligación estatal de administrar el castigo respetando las exigencias de la norma penal sustantiva se conciba de manera objetivista, subjetivista o prospectivista. Lo novedoso de esta segunda conclusión, no reside en las tres formas identificadas de interpretar el Principio. La novedad reside en haber mostrado la vinculación entre estas tres concepciones usuales del Principio de Inocencia con las tres concepciones usuales de obligación moral.

En tercer lugar, se han ofrecido razones a favor de la concepción prospectiva de obligación moral que a su vez sirven para descartar sus contrincantes objetivistas y subjetivistas. Dada la vinculación de esta concepción prospectiva con una manera específica de concebir el Principio de Inocencia, el resultado ha sido ofrecer razones a favor de interpretar el Principio de una manera determinada. Específicamente, el principio debe entenderse como estipulando que el juzgador de los hechos al momento de iniciarse el proceso debe aceptar que no existe ninguna evidencia a favor de la culpabilidad del acusado.

Estas conclusiones son parcialmente independientes entre sí. Puede aceptarse que el modo de concebir la obligación moral incide sobre la manera de interpretar el Principio de Inocencia sin aceptar la manera específica en que el trabajo las vincula, y sin considerar que la interpretación del Principio de Inocencia correlativa con la concepción prospectivista de obligación es la correcta. Adicionalmente, es posible aceptar que las tres interpretaciones 
ofrecidas del Principio de Inocencia efectivamente son correlativas de las tres concepciones de obligación moral y, al mismo tiempo, no aceptar que la concepción prospectivista de obligación, ni su interpretación asociada del Principio, es la adecuada.

\section{BIBLIOGRAFÍA CITADA}

Cárdenas, Raúl (2006): La Presunción de Inocencia (2 edición, México, Porrúa).

Chambers, Henry (1998): "Reasonable Certainty and Reasonable Doubt", Marquette Law Review, vol. n 81 n³: pp. 655-704.

DAmASKA, Mirjan (1986): Faces of Justice and State Authority (New Haven and London, Yale University Press).

Duff, Antony (2013): "Who Must Presume Whom to Be Innocent of What?", Netherlands Journal of Legal Philosophy, vol. 42 n 3: pp. 170-192.

JACKSON, Frank (1991): "Decision-theoretic Consequentialism and the Nearest and Dearest Objection", Ethics, vol. n 101: p. 461-482.

Ladenson, Robert (1980): "In Defense of a Hobbesian Conception of the Law", Philosophy and Public Affairs, vol. 9: pp. 134-159.

Laudan, Larry (2006): Truth, Error and Criminal Law (Cambridge, Cambridge University Press).

MaIer, Julio (1996): Derecho Procesal Penal (Buenos Aires, Editores del Puerto).

ManzinI, Vizenzo (1951): Tratado de Derecho Procesal (Buenos Aires, Ediciones Jurídicas).

Martínez, Zarezka (2005): "Presunción de Inocencia en el Proceso Penal", Justicia y Derecho, n 5: pp. 23-35.

MOORE, George (1912): Ethics (Oxford, Oxford University Press).

Nogueira, Humberto (2005): "Consideraciones Sobre el Derecho Fundamental a la Presunción de Inocencia", Ius et Praxis, vol. 11 n 1: pp. 221-241. 
SELEME, HUGO OMAR: PRESUNCIÓN DE INOCENCIA Y CONCEPCIONES DE LA OBLIGACIÓN MORAL

Prichard, Harold (1949): "Duty and Ignorance of Fact", en Moral Obligation (Oxford, Clarendon Press) pp. 18-39.

Ross, William (1930): The Right and the Good (Oxford, Clarendon Press). (1939): Foundations of Ethics (Oxford, Clarendon Press).

Seleme, Hugo Omar (2013): "Defending the Guilty: A Moral Justification", Ethical Perspectives, vol. $20 \mathrm{n}^{\circ} 2$ : pp. 299-327.

Zimmerman, Michael (2008): Living with Uncertainty (Cambridge, Cambridge University Press).

\section{NORMAS JURIDICAS CITADAS}

Código Procesal Penal del 29 de marzo de 2017.

Constitución española del 27 de diciembre de 1978.

Convención Americana sobre Derechos Humanos del 7 de noviembre de 1969.

Convención Europea de Derechos Humanos del 4 de noviembre de 1950.

Declaración Americana de los Derechos y Deberes del Hombre de 1948.

Declaración Universal de los Derechos Humanos del 10 de diciembre de 1948.

Pacto Internacional de Derechos Civiles y Políticos de 16 de diciembre de 1966.

\section{JURISPRUDENCIA CITADA}

Corte de Apelación de Nebraska (1910) Burnett v. Nebraska, 86 Neb. 11.

Corte del Quinto Circuito, Committee on Pattern Jury Instructions (2015), Pattern Jury Instructions (Criminal Cases). Disponible en: <http://www. Ib5.uscourts.gov/juryinstructions/>, fecha de consulta: 10 febrero 2017.

Corte del Segundo Circuito, General Instructions for Criminal Cases. Disponible en: <http://federalevidence.com/evidence-resources/federaljury-instruction>, fecha de consulta: 10 febrero 2017. 
Corte Suprema de Estados Unidos (1895) Coffin v. United States, 156 U.S. 432.

Corte Suprema de Estados Unidos (1970) In re Winship, 397 U.S. 358.0.

Corte Suprema de Estados Unidos (1990) Cage v. Louisiana, 498 U.S. 39.

Corte Suprema de Estados Unidos (1994) Victor v. Nebraska, 511 U.S. 1.

Corte Suprema del Estado de Massachusetts (1850) Commonwealth v. Webster, 59 Mass. 295-320.

Judicial Council of California (2016), Criminal Jury Instructions CALCRIM, 1, series 1-1800. Disponible en: <http://www.courts.ca.gov/partners/documents/calcrim_2016_edition.pdf>, fecha de consulta: 10 febrero 2017. 